\title{
ADAPTIVE GENERATION OF DECISION FUNCTIONS FOR CLASSIFICATION OF DIGITALLY MODULATED SIGNALS
}

by

\author{
Martin P. DeSimio \\ Air Force Institute of Technology \\ WPAFB, OH 45433
}

\author{
Glenn E. Prescott \\ Air Force Institute of Technology \\ WPAFB, $\mathrm{OH} 45433$
}

\begin{abstract}
This paper describes the performance of an adaptive technique for the generation of decision functions used in classification of several popular types of digitally modulated signals: binary amplitude shift keying (BASK), binary phase shift keying (BPSK), quaternary phase shift keying (QPSK), and binary frequency shift keying (BFSK).

A computer simulation is performed to measure the properties of the classifier which is trained with one signal from each class. This method correctly identifies all the signals considered during this experiment.
\end{abstract}

\section{Background}

Consistent identification of the modulation type of an unknown signals is generally not possible by human operators [1]. Applications such as radio spectrum surveillance and electronic warfare require automatic identification of the modulation type of the received signal $[2,3]$. The first application requires information on modulation type in order to demodulate the signal. The second application uses the information on modulation type in order to choose the appropriate electronic warfare strategy.

The signals considered are limited to forms of digital modulation. Specifically, the modulations are binary amplitude shift keying (BASK), binary phase shift keying (BPSK), quaternary phase shift keying (QPSK), and binary frequency shift keying (BFSK).

The performance of the classification procedure will be investigated by simulations with computer generated signals and noise. This procedure does not attempt to demodulate the unknown signals and is limited to a proof of concept of the proposed method.

A review of unclassified literature from 1982 to 1987 reveals three references concerning the identification of signal modulation type. Two of the papers present similar approaches to the digital modulation identification problem. The earlier paper, by Liedtke, provides the foundation for a paper by Jondral. Both authors employ a technique which synchronizes to the signal baud rate, then calculates frequency, amplitude, and phase estimates to form feature vectors. Liedtke uses intuitive decision functions while Jondral uses an adaptive procedure to generate decision functions. A paper by Chan and others presents an approach for the identification of analog modulation type based upon the mean and variance of the signal envelope.
Several assumptions have been made concerning the signals and the environment. The signals to be processed are assumed to be at the output of the IF amplifier of a receiver, corrupted by additive white gaussian noise. Furthermore, all signals are taken to be at the same carrier frequency and baud rate. Also, it is assumed that only the unknown signal plus noise is present at the output of the IF amplifier. Additionally, the message signal is assumed to have independent and equally likely symbols.

General Structure of Classification Procedure. The classification procedure consists of three steps. The first step is to calculate features from signals having a known modulation type. Each feature is used as a feature vector element. The feature vectors are inputs for the second step, in which these feature vectors are used to generate weight vectors for each signal class using an adaptive algorithm. Thirdly, classification of unknown signals is performed by multiplying feature vectors by weight vectors of each signal class. This results in a linear combination of the elements in the feature vector. This sum is large when the feature and weight vectors are of the same class.

Description of Feature Extraction and Decision Functions. The features used in the classification method are derived from the envelope of the signal and from the spectra of the signal, the signal squared and the signal quadrupled. The mean and variance of the envelope are calculated and used as elements of the feature vector. These features are intended to provide information related to amplitude shift keyed signals.

The magnitude spectrum of the signal is searched for energy of the bandwidth corresponding to the baud rate by correlation with a reference function. Features obtained from this correlation are the magnitude and spectral location of the two largest peaks of the resultant waveform. These elements are intended to provide infomation related to carrier frequency and frequency shift keyed signals.

The magnitude spectra of the signal squared and quadrupled are searched for narrowband energy near twice and four times the carrier frequency. These features are intended to provide information related to the level of phase modulation.

The decision functions used in this experiment are generated using an adaptive technique based on the Least Mean Squares (LMS) algorithm of Widrow and Stearns [4]. The basic processor for the LMS algorithm is the adaptive linear combiner (ALC). An ALC forms a weighted sum of its inputs; the weights on each input line may be modified at each iteration of the algorithm. Based 
upon the input of a known feature vector, the weights in a particular adaptive linear combiner are modified so as to produce the largest sum when input signals are from the class of that feature vector. The procedure adopted here uses an adaptive linear combiner for each class of signal. The class decision for an unknown feature vector is made by choosing the largest output from the set of adaptive linear combiners.

\section{Theory of Operation}

The structure of the processor is shown in Figures 1 and 2 . Figure 1 shows that the nine elements of the feature vectors are obtained from the signal's envelope, the spectrum of the signal, the spectrum of the signal raised to the second power, and the spectrum of the signal raised to the fourth power. Assuming the classifier has been trained, the feature vectors are then used as inputs to the classifier which performs the classification as explained in the previous section.

Eeature Extraction. The features used in this procedure are calculated from four fundamental signal processing operations upon the unknown signal. One of the operations is the calculation of the mean and variance of the envelope of the waveform. Another operation is the searching of the spectrum of the signal for the two largest peaks. The third operation searches the spectrum of the signal squared for peaks near twice the carrier frequency. The fourth operation searches the spectrum of the signal raised to the fourth power for peaks near four times the intermediate frequency.

The physical significance of the feature vector elements are now explained. The first two elements are the mean and variance of the signal envelope. BASK signals have envelopes with a mean less than one-half the amplitude of the carrier while the phase modulated signals have means near the amplitude of the signal. The variance of a BASK signal is greater than that of a phase modulated signal. This is because phase modulated signals have constant envelopes. Therefore, small envelope mean and large envelope variance values indicate BASK while large envelope mean and small envelope variance indicate phase modulated signals.

The following four elements in the feature vector are the spectral location and magnitude of the two largest correlation peaks of the spectrum of the signal with a $\operatorname{sinc}^{2}(x)$ reference function. These provide information related to the location and number of carrier frequencies. One large peak and one much smaller peak indicates a single carrier while two peaks of nearly equal magnitude indicate BFSK.

The results of correlations with spectra of the signal squared and quadrupled provide information related to the level of phase modulation. This technique is based upon the following characteristic: modulation from $M$-ary PSK signals is removed when multiplied by themselves $M$ times [5]. The result of this operation is an unmodulated sinusoid at $\mathrm{M}$ times the original carrier frequency. However, when a signal other than $M$-ary PSK is multiplied by itself $M$ times, its bandwidth will be increased by a factor of $M[6]$.

Normalization of the Feature Vectors. The feature vectors consist of elements whose values may range from on the order of unity to the order of thousands. For example, all the peak correlation values will be less than one, while the spectral location of the correlation peak of the signal raised to the fourth power is above 1600 . This number is the FFT bin number, not a frequency value. In order to prevent this one large element from dominating the adaptation and classification procedure, all feature vector elements are scaled to range from zero to one.

The method used in this experiment to normalize the feature vectors is now explained. The normalization is performed over the sets of signals grouped according to SNR. In practice, the normalization could be performed over the signals collected during one event if off line classification were feasible.

The normalization operates upon the same element of each feature vector at a time. The first element of each feature vector from a particular SNR set is searched for the highest and lowest values. For example, assume the largest element's value is a and the smallest element's value is $b$. The range is found by subtraction to equal a - b. Next, b is subtracted from each element. The result of this subtraction is then divided by the range This normalization provides elements between the values of zero and one for each element of all the feature vectors.

Training. Training the classifier is performed with feature vectors of known class memberships. The training signals in this experiment had $20 \mathrm{~dB}$ signal to noise ratios. One feature vector is calculated from each signal class. These feature vectors are then cyclically applied to the LMS algorithm for a fixed number of iterations.

The output of the algorithm is a weight vector for each class of signal considered. During training, the desired response is a function of the input feature vector and of the weight vectors. This is illustrated in Figure 3.

Feature vectors which have been calculated from known classes are cyclically applied to the algorithm. Weight vectors for each class of signals are updated during each iteration. The procedure is shown in Figure 3 . The weights are updated according to the LMS algortithm as given by Widrow and Stearns [4]. This procedure is applied to feature vectors generated from $20 \mathrm{~dB}$ SNR signals. Convergence of the weight vectors is confirmed by running several trials with different numbers of iterations and different values of the gain constant.

\section{Classification of Unknown Signals}

The unknown signal is processed to generate a feature vector. This feature vector is then multiplied with the four weight vectors. These are the weight vectors calculated by the LMS algorithm during the training. The equations used in this classification process may be written as

$$
d=w_{i}^{T} \cdot x_{j} ; i=1,2,3,4 \text { and } j=1,2,3,4
$$

Class mecobership is determined by selecting the class which corresponds to the weighting function which produces the largest output.

\section{Simulation Results}

The signals are operated upon by the feature extraction and feature vector normalization processes described in previous sections. Feature vectors calculated from the four different classes of signals are presented in Table 1. When these feature vectors are used to train the classifier, the weight vectors of Table 2 are generated. 
The description columns of the tables refer to the feature extraction portion of the experiment. The first and second elements in each feature vector are related to the mean and variance of the envelope. BASK has the smallest mean and largest variance. The third and fourth elements are the result of the correlation of the spectrum of the signal with the $\operatorname{sinc}^{2}(\mathrm{x})$ reference function. The elements correspond to the correlation value and offset to this value, respectively. The fifth and sixth elements of the vectors are similar to the third and fourth, except they are related to the second largest correlation value and its offset. The seventh element is derived from the correlation of the spectrum of the signal squared with the $\operatorname{sinc}^{2}(\mathrm{x})$ reference function. It corresponds to the largest correlation value found near twice the intermediate frequency. The eighth element is similiar to the seventh except it is the result of searching the correlation of the spectrum of the signal raised to the fourth power with the $\operatorname{sinc}^{2}(x)$ function. The value is related to the

largest correlation peak found near four times the intermediate frequency. The ninth element is called the augmentation of the feature vectors. This constant value allows the LMS algorithm to account biases in the feature vectors [4].

The results when the feature vectors are multiplied with the weight vectors are shown in Table 3 . For each class of signal, the largest result of the multiplication occurs when the weight vector is matched to the class of signal.

\section{Conclusions}

The technique presented here uses features which are calculated using conventional signal processing methods. It shows favorable classification properties for the following classes of signals: BASK, BPSK, QPSK, and BFSK. The amount of preprocessing required for feature extraction is comparable to that required by Liedtke and Jondral $[1,3]$. The number of sample points and the observation intervals are comparable between all techniques considered here. The most interesting result is that a new feature useful for the identification of the number of phase states of BPSK and QPSK signals has been shown to provide adequate information identification at SNRs down to $5 \mathrm{~dB}$. These features are the existence of narraowband energy near $\mathrm{M}$ times the carrier frequency for M-ary PSK signals.

However, the conclusiveness of these results is limited due to the small number of trials performed during the simulation.

\section{References}

[1] Liedtke, F.F. "Computer Simulation of an Automatic Classification Procedure for Digitally Modulated Communication Signals with Unknown Parameters "Signal Processing. 6; 311-323 (August 1984).

[2] Chan, Y.T. and others. "Identification of the Modulation Type of a Signal,"Proceedings of the IEEE 1985 International Communications Conference. 22.5.1-22.5.4. New York: IEEE Press, 1985.

[3] Jondral, Freidrich. "Automatic Classification of High Frequency Signals, "Signal Processing. 9: 177-190 (October 1985).

[4] Widrow, Bernard and Samuel D.Stearns. Adaptive Signal Processing. Englewood Cliffs, New Jersey: Prentice-Hall, Inc., 1985.
[5] Proakis, John G. Digital Communications. New York: McGraw-Hill Book Company, 1983.

[6] Gagliardi, Robert M. Introduction to Communications Engineering New York: John Wiley \& Sons, 1978

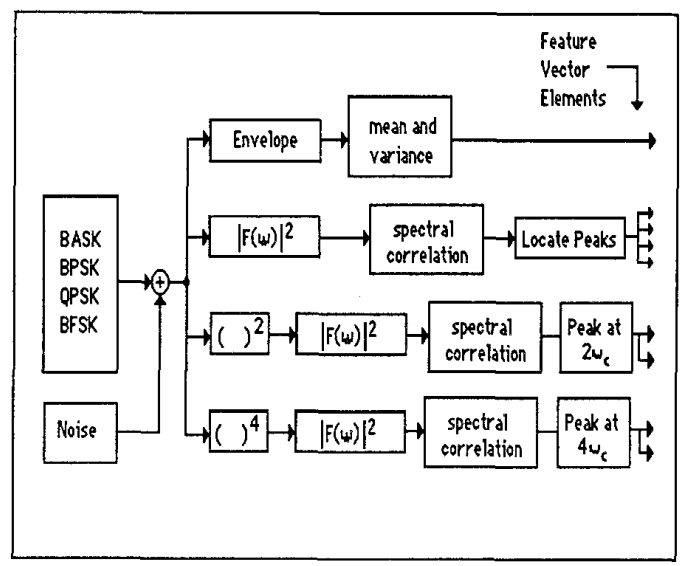

Figure 1. Structure of the Feature Extraction Processing

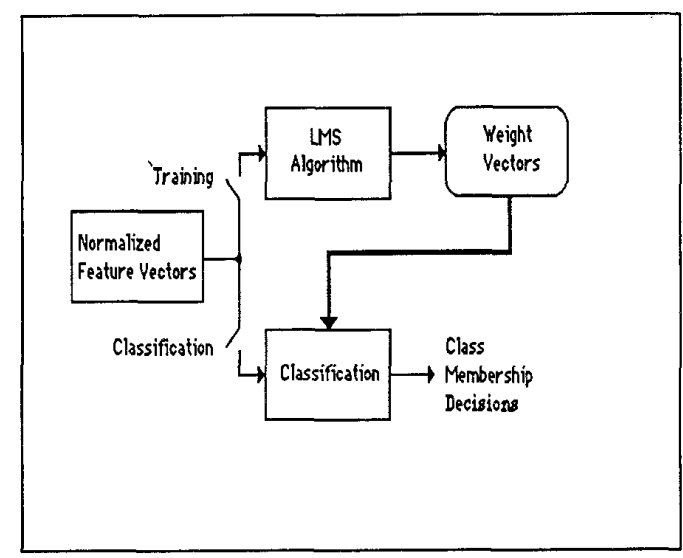

Figure 2. Generation of Weight Vectors and Method of Classification 


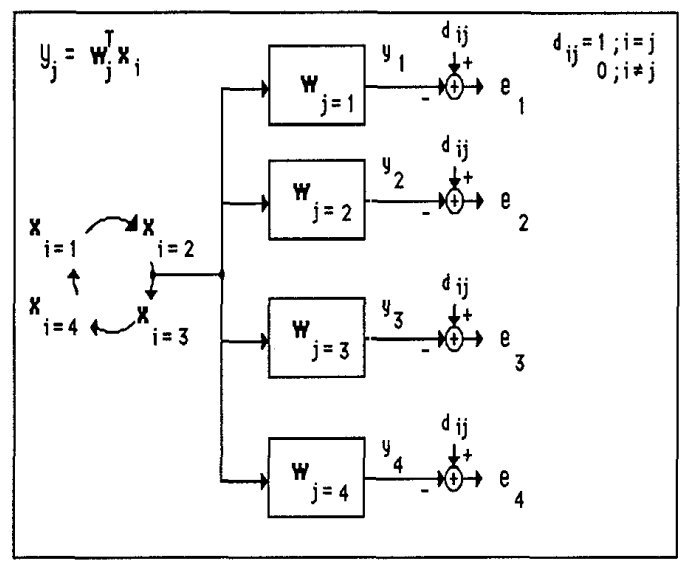

Figure 3. Weight Vector Generation Using the LMS Algorithm

Table 1

Feature Vectors from $20 \mathrm{~dB}$ SNR Signals

$\begin{array}{lrrrr}\text { Description } & \text { BASK } & \text { BPSK } & \text { QPSK } & \text { BFSK } \\ \text { mean } & 0.00000 & 0.99721 & 1.00000 & 0.99400 \\ \text { variance } & 1.00000 & 0.00126 & 0.00429 & 0.00000 \\ \text { maximum 1 } & 1.00000 & 0.04105 & 0.66320 & 0.00000 \\ \text { location 1 } & 1.00000 & 0.97559 & 0.98782 & 0.00000 \\ \text { maximum 2 } & 0.00000 & 0.19540 & 0.06738 & 1.00000 \\ \text { location 2 } & 0.00000 & 0.88890 & 0.94447 & 1.00000 \\ \text { squared } & 0.40009 & 1.00000 & 0.00000 & 0.31237 \\ \text { quadrupled } & 0.00700 & 0.15270 & 1.00000 & 0.00000 \\ \text { augment } & 1.00000 & 1.00000 & 1.00000 & 1.00000\end{array}$

Table 2

Weight Vectors

$\begin{array}{lrrrr}\text { Description } & \text { BASK } & \text { BPSK } & \text { QPSK } & \text { BFSK } \\ \text { mean } & -0.18255 & 0.12045 & 0.13634 & 0.09367 \\ \text { variance } & 0.40205 & -0.19119 & -0.14394 & 0.15369 \\ \text { maximum 1 } & 0.29829 & -0.36294 & 0.21310 & 0.06203 \\ \text { location 1 } & 0.07248 & 0.35930 & 0.10177 & -0.39277 \\ \text { maximum 2 } & 0.10760 & -0.29654 & -0.13564 & 0.56082 \\ \text { location 2 } & -0.15284 & 0.03968 & 0.13853 & 0.15205 \\ \text { squared } & 0.01894 & 0.66547 & -0.40747 & -0.18181 \\ \text { quadrupled } & -0.17228 & -0.18061 & 0.51185 & -0.17937 \\ \text { augment } & 0.22078 & -0.07073 & -0.01114 & 0.25080\end{array}$


Table 3

\begin{tabular}{|c|c|c|c|c|}
\hline & & ication Rest & & \\
\hline & $\mathrm{w}_{1} \mathrm{~T}^{\mathrm{T}}$ & $\mathbf{w}_{2}{ }^{\mathrm{T}} \mathbf{x}$ & $w_{3}{ }^{T} \mathbf{x}$ & $\mathbf{w}_{4}{ }^{\mathrm{T}} \mathbf{x}$ \\
\hline $20 \mathrm{~dB}$ & & & & \\
\hline BASK & 0.8300 & -0.0592 & 0.2167 & 0.1728 \\
\hline BPSK & 0.0875 & 0.9702 & -0.1045 & -0.0272 \\
\hline QPSK & 0.1726 & -0.0862 & 0.8996 & -0.1609 \\
\hline BFSK & 0.3508 & -0.4222 & 0.1772 & 1.0070 \\
\hline $15 \mathrm{~dB}$ & & & & \\
\hline BASK & 0.7107 & -0.0734 & 0.2521 & 0.1771 \\
\hline BPSK & 0.0379 & 0.9505 & 0.0014 & -0.0170 \\
\hline QPSK & -0.0582 & 0.2265 & 0.7370 & -.2281 \\
\hline BFSK & 0.4026 & -.1883 & 0.0012 & 0.9091 \\
\hline $10 \mathrm{~dB}$ & & & & \\
\hline BASK & 0.8544 & -0.0302 & 0.1699 & 0.1641 \\
\hline BPSK & 0.1887 & 0.7744 & 0.1770 & 0.0132 \\
\hline QPSK & -0.1027 & -0.2071 & 0.6016 & 0.3066 \\
\hline BFSK & 0.3266 & 0.2093 & 0.1643 & 0.5617 \\
\hline $5 \mathrm{~dB}$ & & & & \\
\hline BASK & 0.9609 & -0.1639 & 0.0836 & 0.0637 \\
\hline BPSK & 0.0375 & 1.0678 & -0.566 & -0.1530 \\
\hline QPSK & 0.0375 & 0.0 .0125 & 0.9026 & -0.0579 \\
\hline BFSK & 0.1618 & -0.6281 & 0.6975 & 0.9498 \\
\hline
\end{tabular}

